\title{
PROPRIEDADES FÍSICAS DO FEIJÃO DURANTE A SECAGEM: DETERMINAÇÃO E MODELAGEM
}

\author{
Physical properties of edible bean during drying: determination and modelling
}

\author{
Osvaldo Resende ${ }^{1}$, Paulo César Corrêa ${ }^{2}$, André Luís Duarte Goneli ${ }^{3}$ Deise Menezes Ribeiro ${ }^{4}$
}

\begin{abstract}
RESUMO
As propriedades físicas dos produtos agrícolas são influenciadas diretamente pela secagem. Assim, buscou-se com o presente trabalho avaliar o efeito do teor de água nas propriedades físicas dos grãos de feijão da cultivar Vermelho Coimbra, e ajustar diferentes modelos matemáticos aos valores experimentais. Analisaram-se duas metodologias para a determinação da massa específica aparente e da massa específica unitária. A massa específica aparente e a porosidade da massa foram determinadas utilizando-se uma balança de peso hectolitro e um picnômetro de comparação a ar, respectivamente. A massa específica unitária foi determinada em função da porosidade e da massa específica aparente. Os dados experimentais da massa específica aparente e unitária foram comparados com os obtidos pelo cálculo a partir da composição química média dos grãos de feijão. Concluiu-se que a redução no teor de água pela secagem influencia as propriedades físicas dos grãos, proporcionando a diminuição da porosidade e o aumento das massas específicas aparente e da massa específica unitária. A metodologia de determinação das massas específicas pela composição química média dos grãos de feijão apresenta limitação quanto à precisão dos resultados. As variações das propriedades físicas analisadas em função do teor de água são adequadamente representadas pelo modelo linear.
\end{abstract}

Termos para indexação: Phaseolus vulgaris, porosidade, massa específica, modelos matemáticos.

\section{ABSTRACT}

The physical properties of the agricultural products are influenced directly by drying. Like this, the objective of this work was to evaluate the effect of moisture content variation in the physical properties of edible bean grain Vermelho Coimbra cultivar, and to fit different mathematical models to the experimental values. Two methodologies were analyzed for the determination of the bulk density and true density. The bulk density and the porosity were determined being used a hectoliter balance and a picnometer, respectively. The true density was determined in function of the porosity and the bulk density. The experimental data of the bulk density and true density were compared to the obtained data by the calculation starting from the chemical composition of the edible beans. It was concluded that the moisture content reduction affects the the physical properties of edible bean grain, providing the decrease of the porosity and of the mass of 1000 grains and the increase of bulk density and true density. The methodology of determination of the bulk density and true density using the chemical composition of the bean grains presents limitation with relationship to the precision of the results. The variations of the physical properties analyzed in function of the moisture content are better fitted represented by the lineal model.

Index terms: Phaseolus vulgaris, porosity, bulk density, mathematical models.

\section{(Recebido em 19 de julho de 2005 e aprovado em 31 de outubro de 2006}

\section{INTRODUÇÃO}

O feijoeiro comum (Phaseolus vulgaris L.) é cultivado em quase todos os países de clima tropical e subtropical assumindo grande importância na alimentação humana, devido, fundamentalmente, ao seu baixo custo para o consumidor e por ser considerado um alimento balanceado nutricionalmente. É a principal fonte de proteína na alimentação da população brasileira, sendo utilizado em substituição a carnes e outros produtos protéicos, formando juntamente com o arroz, uma base alimentar (FERNANDES \& SOUZA FILHO, 2001; RIOS et al., 2003).

O mercado mundial de feijão movimenta, anualmente, mais de dezoito milhões de toneladas, sendo o Brasil o segundo maior produtor e consumidor (AGRIANUAL, 2003). Na safra 2003/2004 a produção brasileira de feijão foi de três milhões de toneladas, apresentando uma produtividade média de $700 \mathrm{~kg} \mathrm{ha}^{-1}$ (CONAB, 2005).

\footnotetext{
1Doutor em Engenharia Agrícola, Professor do Centro Federal de Educação Tecnológica/CEFET-RV - Rodovia Sul Goiana, Km 1, Zona Rural Rio Verde, GO - osvresende@yahoo.com.br

${ }^{2}$ Engenheiro Agrônomo, Dr., Professor Adjunto do Departamento de Engenharia Agrícola da Universidade Federal de Viçosa/UFV - Campus UFV Cx. P. 270 - 36570-000 - Viçosa, MG - copace@ufv.br

${ }^{3}$ Engenheiro Agrônomo, Doutorando no Departamento de Engenharia Agrícola da Universidade Federal de Viçosa/UFV - Campus UFV - Cx. P. 270 36570-000 - Viçosa, MG - andregoneli@yahoo.com.br

${ }^{4}$ Engenheira Agrícola, Doutora - CONAB - Av. Princesa Isabel, 629 conjunto 702 - 29010-904 - Vitória, ES - deise.ribeiro@conab.gov.br
} 
A secagem do feijão é o processo mais utilizado para assegurar sua qualidade e estabilidade, uma vez que as atividades biológicas e físico-químicas que ocorrem durante o armazenamento, diminuem com a redução do teor de água dos grãos.

A redução do teor de água dos grãos influencia diretamente na alteração das suas propriedades físicas durante a secagem. Desta forma, a correta determinação das propriedades físicas é de importância na otimização dos processos industriais, estudos de aerodinâmica, projeto e dimensionamento de equipamentos utilizados nas operações de colheita e pós-colheita.

Pesquisadores têm realizado estudos para a avaliação das principais propriedades físicas dos produtos agrícolas, demonstrando a sua aplicação prática em projetos de máquinas e estruturas. Recentes descobertas científicas têm melhorado a manipulação e o processamento dos materiais biológicos, com a utilização de processos mecânicos, térmicos, elétricos, ópticos, dentre outros, mas pouco é conhecido sobre as características físicas dos produtos (AMIN et al., 2004).

Autores têm investigado as variações das propriedades físicas em função do teor de água e de outros fatores durante a secagem para diversos produtos como para trigo e canola (LANG \& SOKHANSANJ, 1993), batata desidratada (MCMINN \& MAGEE, 1997), milho pipoca (RUFFATO et al., 1999), lentilha (AMIN et al., 2004), semente de abóbora (PAKSOY \& AYDIN, 2004), milheto (BARYEH, 2002), semente de quiabo (SAHOO \& SRIVASTAVA, 2002), noz de caju (BALASUBRAMANIAN, 2001), semente de algodão (OZARSLAN, 2002), ervilha (BARYEH \& MANGOPE, 2002), café (CHANDRASEKAR \& VISWANATHAN, 1999) e cacau (BART-PLANGE \& BARYEH, 2003).

Considerando a importância do assunto e variabilidade existente nas propriedades físicas dos produtos agrícolas de diferentes espécies e variedades, foi realizado este trabalho com o objetivo de determinar a massa de 1000 grãos, a porosidade e as massas específicas aparente e unitária dos grãos de feijão ao longo do processo de secagem, bem como ajustar modelos matemáticos aos valores experimentais. Também objetivou-se analisar diferentes metodologias de determinação da massa específica aparente e da massa específica unitária.

\section{MATERIAL E MÉTODOS}

O trabalho foi desenvolvido em outubro de 2003 no Laboratório de Propriedades Físicas e Qualidade de Produtos Agrícolas pertencente ao Centro Nacional de
Treinamento em Armazenagem (CENTREINAR) da Universidade Federal de Viçosa (UFV), Viçosa-MG.

Foram utilizados grãos de feijão da cultivar Vermelho Coimbra, grupo comercial vermelho, cultivados no município de Cajuri-MG e colhidos, manualmente, com teor de água de aproximadamente 0,45 base seca (decimal b.s.).

Posteriormente, o produto foi submetido à secagem em estufa com ventilação forçada, mantida à temperatura de $40{ }^{\circ} \mathrm{C}$. A redução do teor de água ao longo de secagem foi acompanhada pelo método gravimétrico (perda de massa), conhecendo-se o nível de umidade inicial do produto, até atingir o teor de água final de 0,13 (b.s.), com auxílio de uma balança analítica com precisão de $0,01 \mathrm{~g}$.

Os teores de água do feijão foram determinados pelo método de estufa, a $105 \pm 1{ }^{\circ} \mathrm{C}$, até massa constante. Para cada teor de água obtido, o produto foi homogeneizado e encaminhado para determinação das propriedades físicas.

\section{Porosidade (ع)}

A porosidade da massa de feijão foi determinada, pela média de três repetições, utilizando um picnômetro de comparação a ar, segundo o processo descrito por Day, citado por Mohsenin (1986).

\section{Massa específica aparente $\left(\rho_{a p}\right)$ e unitária $\left(\rho_{u}\right)$}

A massa específica aparente $\left(\rho_{\mathrm{ap}}\right)$, expressa em $\mathrm{kg}$ $\mathrm{m}^{-3}$, foi determinada utilizando-se uma balança de peso hectolitro, com capacidade de um litro, em três repetições para cada teor de água.

A massa específica unitária $\left(\rho_{\mathrm{u}}\right)$, expressa em $\mathrm{kg} \mathrm{m}^{-3}$, foi obtida em função da porosidade e da massa específica aparente de acordo com a equação descrita por Mohsenin (1986):

$$
\rho_{\mathrm{u}}=\frac{\rho_{\mathrm{ap}}}{(1-\varepsilon)}
$$

em que:

$\left(\rho_{\text {ap }}\right)$ : massa específica aparente, $\mathrm{kg} \mathrm{m}^{-3}$;

$\left(\rho_{\mathrm{u}}\right)$ : massa específica unitária, $\mathrm{kg} \mathrm{m}^{-3}$;

$\varepsilon$ : porosidade, $\%$.

Os resultados das massas específicas aparente e unitária foram comparados aos obtidos pelo cálculo da composição química média dos grãos de feijão, adaptando a metodologia descrita por Lewis (1993), de acordo com a seguinte expressão: 


$$
\rho=\frac{1+\mathrm{U}^{*}}{\left(\mathrm{U}^{*} / \rho_{\mathrm{a} g}\right)+\left(\omega_{1} / \rho_{1}\right)+\cdots+\left(\omega_{\mathrm{n}} / \rho_{\mathrm{n}}\right)}
$$

em que:

$\rho$ : massa específica, $\mathrm{kg} \mathrm{m}^{-3}$

$\rho_{\mathrm{ag}}$ : massa específica da água, $1000 \mathrm{~kg} \mathrm{~m}^{-3}$

$\mathrm{U}^{*}$ : teor de água do produto, decimal (base seca)

$\rho_{1} \ldots \rho_{\mathrm{n}}$ : massa específica dos constituintes de 1 a n, $\mathrm{kg} \mathrm{m}^{-3}$

$\omega_{1} \ldots \omega_{\mathrm{n}}$ : teores dos constituintes do produto de $1 \mathrm{a} \mathrm{n}$, decimal (base seca)

Foram utilizados os teores médios dos componentes dos grãos de feijão segundo a USDA (1975), conforme a Tabela 1.

Tabela 1 - Composição química média dos grãos de feijão em 100 gramas do produto e massa específica dos componentes.

\begin{tabular}{lcc}
\hline Constituinte & $\begin{array}{c}\text { Participação } \\
\text { na composição } \\
\text { química }(\mathrm{mg})\end{array}$ & $\begin{array}{c}\text { Massa } \\
\text { específica dos } \\
\text { constituintes } \\
\left(\mathrm{kg} \mathrm{m}^{-3}\right)\end{array}$ \\
\hline Água & $16.540,0$ & 1.000 \\
Proteína & $20.700,0$ & 1.400 \\
Carboidratos & $53.890,0$ & 1.560 \\
(Totais) & 9,846 & - \\
Vitaminas & $1.742,0$ & 900 \\
Lipídios & & \\
(Totais) & 107,0 & 1.580 \\
Minerais & 401,0 & 2.340 \\
Cálcio & 5,15 & 7.865 \\
Fósforo & $1.413,0$ & 860 \\
Ferro & 0,788 & 8.875 \\
Potássio & 1,167 & 7.400 \\
Cobre & 176,0 & 1.714 \\
Manganês & 17,0 & 970 \\
Magnésio & 2,56 & 7.177 \\
Sódio & & \\
Zinco & & \\
\hline
\end{tabular}

\section{Massa de 1000 grãos (m)}

A massa de 1000 grãos foi determinada para cada teor de água ao longo da secagem utilizando-se uma balança eletrônica com precisão de $0,01 \mathrm{~g}$, em três repetições, sendo os resultados expressos em quilograma $(\mathrm{kg})$.

\section{Análise Estatística}

Os dados obtidos da porosidade, massa de 1000 grãos, massa específica unitária e massa específica aparente foram submetidos à análise de regressão. Os modelos foram escolhidos com base na significância da equação, pelo teste $\mathrm{F}$, e dos coeficientes de regressão ( $\beta$ 's), utilizando-se o teste "t", a fim de se estabelecerem equações que representem as interações entre as variáveis analisadas.

\section{RESULTADOS E DISCUSSÃO}

Na Figura 1, são apresentados os valores experimentais e estimados da porosidade da massa de grãos de feijão em função do teor de água (b.s.).

A porosidade da massa de grãos apresentou para o feijão valores entre 44,1 a $38,3 \%$, na faixa de umidade estudada. Observa-se que a porosidade descreve comportamento semelhante à maioria dos produtos agrícolas, apresentando redução dos seus valores com a diminuição do teor de água. O modelo linear representa satisfatoriamente aos valores experimentais, sendo significativo a $1 \%$ de probabilidade pelo teste " $t$ ", e apresentando elevado valor do coeficiente de determinação $\left(\mathrm{R}^{2}\right)$. Afonso Júnior et al. (2000) também constataram o mesmo comportamento linear para a porosidade dos grãos de milheto durante a secagem, embora estes tenham apresentado maiores valores de porosidade comparados com o feijão. Este fato, provavelmente, é devido ao grão de feijão apresentar maior tamanho em relação ao milheto, o que, para um mesmo volume, proporciona uma menor quantidade de espaços intergranulares.

Nas Figuras 2 e 3, são apresentados os valores experimentais e estimados da massa específica aparente e unitária e os valores calculados em função da composição química do feijão, para os diferentes teores de água.

Conforme as Figuras 2 e 3, verifica-se que, independentemente da metodologia utilizada, houve redução da massa específica aparente e da massa específica unitária dos grãos de feijão com o aumento do teor de água. Os valores experimentais da massa específica aparente e unitária para o feijão variaram entre 893 a $761 \mathrm{~kg}$ $\mathrm{m}^{-3}$ e 1468 a $1361 \mathrm{~kg} \mathrm{~m}^{-3}$, respectivamente, para o teor de água do produto variando de 0,42 a 0,11 (b.s.). Estes resultados são coerentes com a maioria dos produtos agrícolas pesquisados (AFONSO JÚNIOR et al., 2000; CHANDRASEKAR \& VISWANATHAN, 1999; CORRÊA et al., 2004; RUFFATO et al., 1999). 


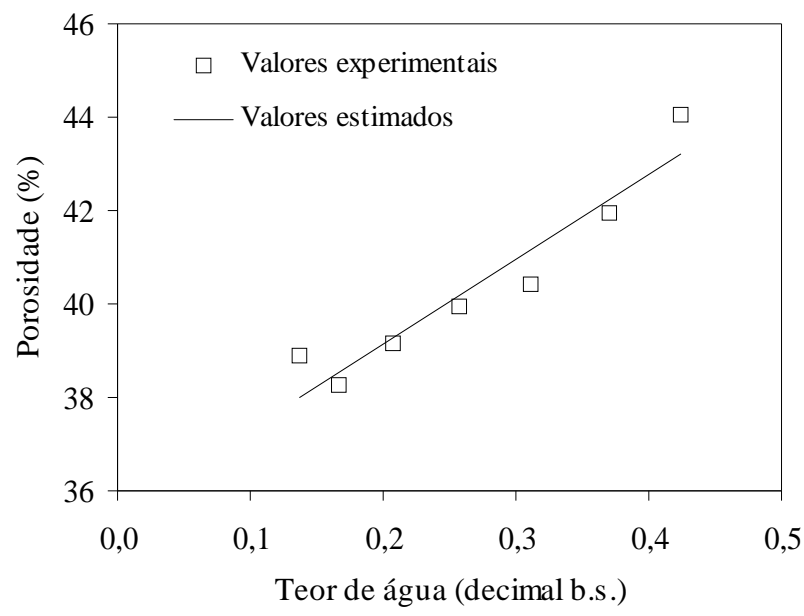

Figura 1 - Valores experimentais e estimados da porosidade $(\varepsilon)$ da massa de feijão em função do teor de água $\left(U^{*}\right)$.

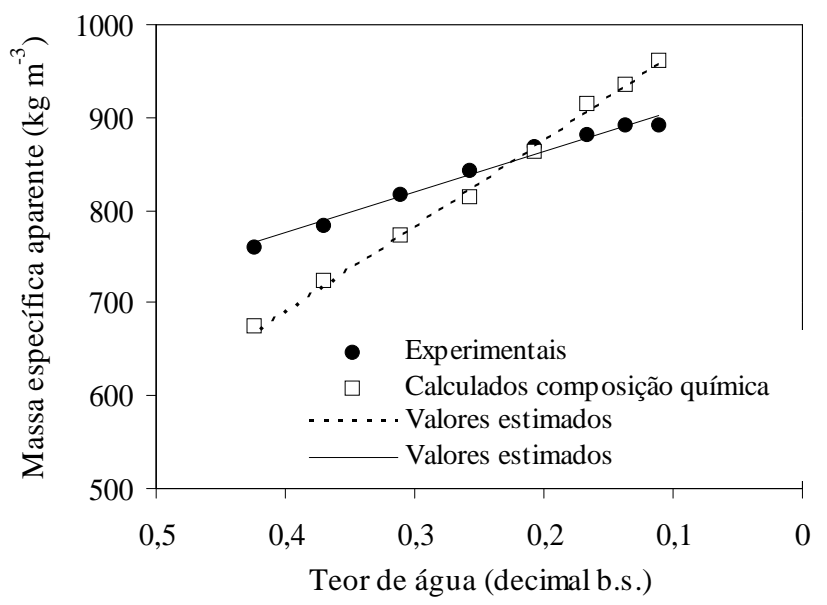

Figura 2 - Valores experimentais da massa específica aparente e calculados em função da composição química dos grãos de feijão para diferentes teores de água.

Os valores das massas específicas, obtidos em função da composição química do feijão, foram superiores aos do experimento até o teor de água ao redor de 0,22 (b.s.). Acima desse limite os valores das massas específicas foram subestimados pelo cálculo utilizando a composição química dos componentes do feijão. Corrêa et al. (2004) calcularam as massas específicas aparente e unitária utilizando os valores da composição química dos grãos de trigo e constataram que os valores estimados foram inferiores aos dados experimentais obtidos. Por outro lado, Afonso Júnior et al. (2000) e Lewis (1993), utilizando desta mesma metodologia para determinação da massa específica unitária do milheto e da maçã, respectivamente, obtiveram resultados superiores aos obtidos experimentalmente. Assim, de acordo com os resultados encontrados na literatura e no presente trabalho, deve-se ressaltar que esta metodologia de determinação das massas específicas incorpora valores da composição química média do produto e apresenta discrepâncias nos resultados.

Na Tabela 2 são apresentados os modelos lineares de regressão ajustados aos valores da massa específica aparente e massa específica unitária (experimentais e calculados pela composição química do produto) e os coeficientes de determinação $\left(\mathrm{R}^{2}\right)$ com seus respectivos níveis de significância. Observa-se que os valores experimentais da massa específica aparente e da massa específica unitária, bem como os valores calculados com base na participação de cada componente químico do feijão podem ser, satisfatoriamente, representados pelo modelo linear devido ao elevado valor do $\mathrm{R}^{2}$. Os coeficientes dos modelos lineares ajustados aos dados experimentais apresentaram elevada significância, comprovando que a massa específica do feijão decresce linearmente com o aumento do teor de água. O mesmo comportamento linear para a variação das massas específicas aparente e unitária durante a secagem foi constatado por Chandrasekar \& Viswanathan (1999) em café, Afonso Júnior et al. (2000) para o milheto, Aydin (2003) para amêndoa e Corrêa et al. (2004) para o trigo.

O efeito da variação do teor de água na massa de 1000 grãos de feijão é apresentado na Figura 4. Verificase que com a redução do teor de água de 0,45 a 0,11 (b.s.), a massa de 1000 grãos variou de 0,31 a $0,23 \mathrm{~kg}$.

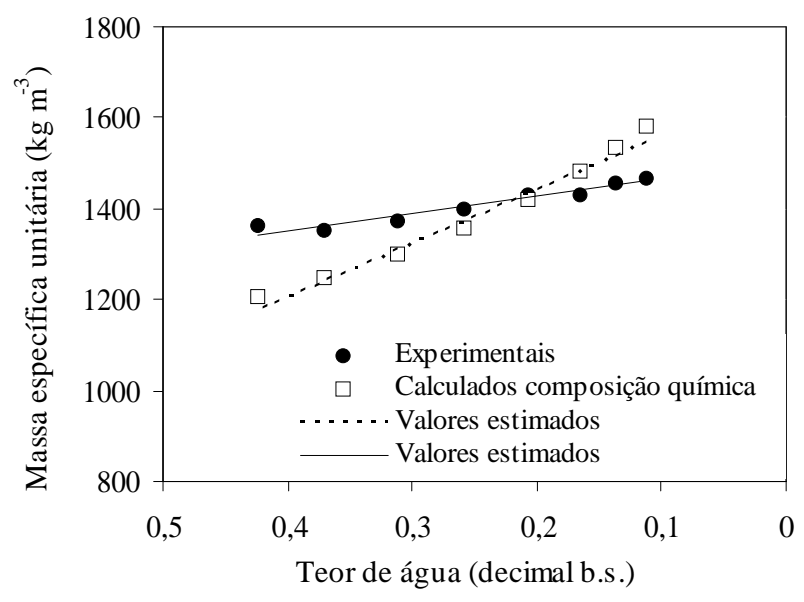

Figura 3 - Valores experimentais da massa específica unitária em função da porosidade e massa específica aparente e calculados pela composição química dos grãos de feijão para diferentes teores de água. 
O modelo linear representa adequadamente os dados experimentais, indicado pela significância a $1 \%$ de probabilidade pelo teste " $t$ ", e elevado valor do coeficiente de determinação $\left(\mathrm{R}^{2}\right)$. Estes resultados corroboram os outros autores que encontraram valores semelhantes para diversos produtos agrícolas (AKINCI et al., 2004; AMIN et al., 2004; BART-PLANGE \& BARYEH, 2003; BARYEH, 2001, 2002; KALEEMULLAH \& GUNASEKAR, 2002).

Tabela 2 - Modelos de regressão ajustados aos valores experimentais obtidos de massa específica aparente $\left(\rho_{\text {apl }}\right)$ e calculados em função da sua composição química $\left(\rho_{\text {ap } 2}\right)$; valores experimentais de massa específica unitária $\left(\rho_{\mathrm{u} 1}\right)$ e calculados em função da composição química $\left(\rho_{\mathrm{u} 2}\right)$; coeficientes de determinação $\left(\mathrm{R}^{2}\right)$ com seus respectivos níveis de significância.

\begin{tabular}{lc}
\hline Equações de regressão & $\mathrm{R}^{2}(\%)$ \\
\hline Massa específica aparente & $99,42^{* *}$ \\
$\rho_{\mathrm{ap} 1}=960,26-467^{++} \cdot\left(\mathrm{U}^{*}\right)$ & $99,68^{* *}$ \\
$\rho_{\mathrm{ap} 2}=1059,1-915,4^{++} \cdot\left(\mathrm{U}^{*}\right)$ & \\
\hline Massa específica unitária & $91,98^{* *}$ \\
$\rho_{\mathrm{u} 1}=1496,9-362,1^{++} \cdot\left(\mathrm{U}^{*}\right)$ & $98,36^{* *}$ \\
$\rho_{\mathrm{u} 2}=1665,8-1134,2^{++} \cdot\left(\mathrm{U}^{*}\right)$ &
\end{tabular}

$\mathrm{U}^{*}$ : teor de água (decimal b.s.)

** Significativo a $1 \%$ de probabilidade, pelo teste $\mathrm{F}$.

${ }^{++}$Significativo a $1 \%$ de probabilidade, pelo teste $\mathrm{t}$.

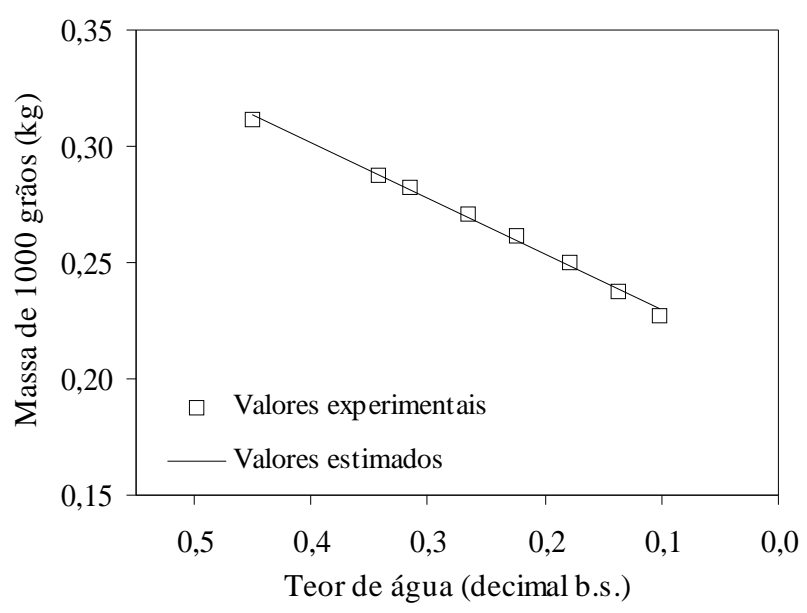

Figura 4-Valores experimentais e estimados da massa de 1000 grãos (m) para o feijão, em função da redução do teor de água.

\section{CONCLUSÕES}

Com base nos resultados obtidos pode-se concluir que:

A redução do teor de água influencia as propriedades físicas dos grãos de feijão da cultivar Vermelho Coimbra, proporcionando a diminuição da porosidade e o aumento das massas específicas aparente e da massa específica unitária.

O modelo linear representa adequadamente as propriedades físicas dos grãos de feijão analisados;

Os valores das massas específicas unitária e aparente obtidos em função da composição química do produto, foram superiores aos dados experimentais até o teor de água de 0,22 (b.s.), acima deste limite os valores das massas específicas foram subestimados;

A metodologia de determinação das massas específicas utilizando a composição química média dos grãos de feijão, apresenta limitação quanto à precisão dos resultados.

\section{AGRADECIMENTOS}

Os autores agradecem à CAPES, ao CNPq e a FAPEMIG, pelo apoio financeiro indispensável na execução do presente trabalho.

\section{REFERÊNCIAS BIBLIOGRÁFICAS}

AFONSO JÚNIOR, P. C.; CORRÊA, P. C.; ANDRADE, E. T. Análise da variação das propriedades físicas e contração volumétrica dos grãos de milheto (Pennisetum glaucum) durante o processo de dessorção. Revista Brasileira de Armazenamento, Viçosa, v. 25, n. 1, p. 15-21, 2000.

AGRIANUAL. Anuário da agricultura brasileira. São Paulo: Comércio \& Consultoria, 2003. 544 p.

AKINCI, I.; OZDEMIR, F.; TOPUZ, A.; KABAS, O.; CANAKCI, M. Some physical and nutritional properties of Juniperus drupacea fruits. Journal of Food Engineering, London, v. 65, p. 325-331, 2004.

AMIN, M. N.; OSSAIN, M. A.; ROY, K. C. Effects of moisture content on some physical properties of lentil seeds. Journal of Food Engineering, London, v. 65, p. 83-87, 2004.

AYDIN, C. Physical properties of almond nut and kernel. Journal of Food Engineering, London, v. 60, p. 315-320, 2003.

BALASUBRAMANIAN, D. Physical properties of raw cashew nut. Journal Agricultural Engineering Research, [S.1.], v. 78, n. 3, p. 291-297, 2001. 
BART-PLANGE, A.; BARYEH, E. A. The physical properties of category B cocoa beans. Journal of Food Engineering, London, v. 60, p. 219-227, 2003.

BARYEH, E. A. Physical properties of bambara groundnuts. Journal of Food Engineering, London, v. 47, p. 321-326, 2001.

BARYEH, E. A. Physical properties of millet. Journal of Food Engineering, London, v. 51, p. 39-46, 2002.

BARYEH, E. A.; MANGOPE, B. K. Some physical properties of QP-38 variety pigeon pea. Journal of Food Engineering, London, v. 51, p. 39-46, 2002.

CHANDRASEKAR, S. V.; VISWANATHAN, R. Physical and thermal properties of coffee.

Journal Agricultural Engineering Research, [S.1.], v. 73, p. 227-234, 1999.

COMPANHIA NACIONAL DE ABASTECIMENTO. CONAB. Disponível em: <www.conab.gov.br>. Acesso em: 10 jan. 2005.

CORRÊA, P. C.; RIBEIRO, D. M.; RESENDE, O.; HENRIQUES, D. R.; SOUZA, M. A. Análise da porosidade, massa específica aparente e real do trigo durante o processo de secagem. In: CONGRESSO BRASILEIRO DE ENGENHARIA AGRÍCOLA, 33., 2004, São Pedro, SP. Anais... São Pedro: SBEA, 2004. CD-ROM.

FERNANDES, G. M. B.; SILVEIRA FILHO, B. F. Armazenamento de sementes de feijão na pequena propriedade. Rio de Janeiro: PESAGRO, 2001. 5 p.

KALEEMULLAH, S.; GUNASEKAR, J. J. Moisturedependent physical properties of arecanut kernels. Biosystems Engineering, London, v. 82, n. 3, p. 331-338, 2002.
LANG, W.; SOKHANSANJ, S. Bulk volume shrinkage during drying of wheat and canola. Journal of Food Process Engineering, Trumbull, v. 16, n. 4, p. 305-314, 1993.

LEWIS, M. J. Propriedades físicas de los alimentos y de los sistemas de procesados. Zaragoza: Acribia, 1993. $494 \mathrm{p}$.

MCMINN, W. A. M.; MAGEE, T. R. A. Physical characteristics of dehydrated potatoes: part I. Journal of Food Engineering, London, v. 33, n. 1/2, p. 37-48, 1997.

MOHSENIN, N. N. Physical properties of plant and animal materials. New York: Gordon and Breach, 1986. 841 p.

PAKSOY, M.; AYDIN, C. Some physical properties of edible squash (Cucurbita pepo L.) seeds. Journal of Food Engineering, London, v. 65, p. 225-231, 2004.

OZARSLAN, C. Physical properties of cotton seed. Biosystems Engineering, [S.1.], v. 83, n. 2, p. 169-174, 2002.

RIOS, A. O.; ABREU, C. M. P.; CORRÊA, A. D. Efeito da estocagem e das condições de colheita sobre algumas propriedades físicas, químicas e nutricionais de três cultivares de feijão (Phaseolus vulgaris L.). Ciência e Tecnologia de Alimentos, Campinas, v. 23, p. 39-45, 2003.

RUFFATO, S.; CORRÊA, P. C.; MARTINS, J. H.; MANTOVANI, B. H. M.; SILVA, J. N. Influência do processo de secagem sobre a massa específica aparente, massa específica unitária e porosidade de milho-pipoca. Revista Brasileira de Engenharia Agrícola e Ambiental, Campina Grande, v. 3, n. 1, p. 45-48, 1999.

SAHOO, P. K.; SRIVASTAVA, A. P. Physical properties of okra seed. Biosystems Engineering, [S.1.], v. 84, n. 4, p. 441-448, 2002.

UNITED STATES DEPARTMENT OF AGRICULTURE. Handbook of the nutritional contents of foods. New York: Dover, 1975. $190 \mathrm{p}$. 